\title{
ON REARRANGING MAXIMAL FUNCTIONS IN $\mathbf{R}^{n}$
}

\author{
by P. L. WALKER \\ (Received 27th February 1974)
}

\section{Introduction}

Denote by $f$ a positive measurable function on $\mathbf{R}^{n}$, and by $\lambda$ the distribution function of $f: \lambda(y)=|\{x: f(x)>y\}|$, where $y>0$, and $|\{x: \ldots\}|$ denotes the Lebesgue measure of the set specified. We shall suppose that $\lambda(y)<\infty$ for each $y>0$, and that $\lambda(y) \rightarrow 0$ as $y \rightarrow \infty$. The decreasing rearrangement $f^{*}$ of $f$ is defined on $(0, \infty)$ by

$$
f^{*}(t)=\inf \{y: \lambda(y) \leqq t\}, \quad 0<t<\infty
$$

The class $L^{*}\left(\mathbf{R}^{n}\right)$ comprises those functions $f$ for which $\int_{0}^{1} f^{*}(t) d t<\infty$ and $f^{*}(t) \rightarrow 0$ as $t \rightarrow \infty$ : equivalently $f \in L^{*}\left(\mathbf{R}^{n}\right)$ if and only if for each $c>0$,

$$
f \chi_{\{x: f(x)>c\}} \in L^{1}\left(\mathbf{R}^{n}\right) \text {. }
$$

The averaged rearrangement $f^{* *}$ is defined on $(0, \infty)$ by

$$
f^{* *}(t)=t^{-1} \int_{0}^{t} f^{*}(u) d u
$$

It is continuous on $(0, \infty)$, decreasing, and tends to zero as $t \rightarrow \infty$.

For a bounded open set $K \subset \mathbf{R}^{n}$, which we suppose to be convex and symmetric with respect to the origin, the Hardy-Littlewood maximal function ${ }^{7} m f$ is defined at each $x \in \mathbf{R}^{n}$ by

$$
m f(x)=\sup _{\lambda>0}\left(\lambda^{n}|K|\right)^{-1} \int_{x+\lambda K} f,
$$

where the integration is with respect to Lebesgue measure. In $\mathbf{R}^{1}, m f(x)$ reduces to $\sup _{\lambda>0}(2 \lambda)^{-1} \int_{x-\lambda}^{x+\lambda} f$.

The object of this paper is to show that the functions $(m f)^{*}$ and $f^{* *}$ are of the same order of magnitude, a typical result being that

$$
2^{-n-1} f^{* *}\left(2^{n} t\right) \leqq(m f)^{*}(t) \leqq f^{* *}\left(2^{-n} t\right) .
$$

The special case where $K$ is the unit cube has been considered by C. S. Herz and the author in (2) and (6) where less precise constants are obtained.

If in $L^{*}\left(\mathbf{R}^{1}\right)$ we replace $m f$ by $\theta f=\sup _{\lambda>0} \lambda^{-1} \int_{x-\lambda}^{x} f$, there is the well-known result of F. Riesz (3) that $(\theta f)^{*}(t) \leqq f^{* *}(t)$. However, the corresponding result 
(without the intervention of any constant) for $m f$ is not valid, as we show in Section 3.

Estimates for the maximal function are of importance for their application s to the theories of differentiation and singular integrals as appears from (1) and (4).

\section{Principal results}

Theorem. Let $f \in L^{*}\left(\mathbf{R}^{n}\right)$, and $m f, f^{* *}$ be as defined in Section 1 .

Then for each $t>0,(m f)^{*}(t) \leqq f^{* *}\left(2^{-n} t\right)$,

and if $A$ is a positive constant,

$$
f^{* *}(A t) \leqq B(m f)^{*}(t),
$$

where $B=2^{n}\left(1+2^{n} / A\right)$ if $A<2^{n}$, and $B=\left(1+\left(2^{n} / A\right)^{1 /(n+1)}\right)^{n+1}$ if $A \geqq 2^{n}$.

The proof of this theorem requires some standard facts about rearrangements which are listed as Lemma 1, and whose proof may be found for instance in (5), page 201, lemma 3.17, and also some simple geometrical results on convex sets which are stated (and proved) as Lemma 2.

Lemma 1. (i) For any measurable set $E \subset \mathbf{R}^{n}$,

$$
\int_{E} f \leqq \int_{0}^{|E|} f^{*}
$$

(ii) For any $t>0, f^{* *}(t)=\sup \left\{\frac{1}{|F|} \int_{F} f: t \leqq|F|<\infty\right\}$.

Lemma 2. Let $K$ be a bounded open set in $\mathbf{R}^{n}$ which is convex and symmetric about the origin.

(i) If $0<\lambda \leqq 1$, and $K \cap(x+\lambda K) \neq \varnothing$, then $x \in 2 K$.

(ii) If $y \in x+\alpha \lambda K$, where $\alpha, \lambda>0$, then $y+(1+\alpha) \lambda K \supset x+\lambda K$.

(iii) Let $H$ be a bounded subset of $\mathbf{R}^{n}$, and $\Lambda$ a finite subset of $(0, \infty)$. Suppose that for each $x \in H$, we are given a value of $\lambda=\lambda(x) \in \Lambda$. Then we can choose $a$ finite number of points $x_{1}, x_{2}, \ldots, x_{m}$ of $H$ so that the sets $\left(x_{i}+\lambda\left(x_{i}\right) K\right)_{i=1}^{m}$ are disjoint, while the sets $\left(x_{i}+2 \lambda\left(x_{i}\right) K\right)_{i=1}^{m}$ cover $H$.

Proof. (i) Let $z \in K \cap(x+\lambda K)$, where $0<\lambda \leqq 1$.

Then $z=x+\lambda k$ where $k \in K$, and so $-k \in K$ since $K$ is symmetric. It follows that $x=(1+\lambda)\left(\frac{1}{1+\lambda} z+\frac{\lambda}{1+\lambda}(-k)\right) \in(1+\lambda) K \subset 2 K$.

This proves (i) and the proof of (ii) is similar. $\alpha>0$.

(iii) We denote $x_{i}+\lambda\left(x_{i}\right) K$ by $K\left(x_{i}\right)$ and $x_{i}+\alpha \lambda\left(x_{i}\right) K$ by $K_{\alpha}\left(x_{i}\right)$ for a given 
Since there are only a finite number of choices possible for $\lambda\left(x_{i}\right)$, we choose as $x_{1}$ a point for which $\lambda\left(x_{i}\right)$ is as large as possible. Having chosen $x_{1} \ldots x_{r}$ say, we choose $x_{r+1}$ from among those points $x$ of $H$ for which $K(x)$ is disjoint from $K\left(x_{1}\right) \cup \ldots \cup K\left(x_{r}\right)$, and $\lambda\left(x_{r+1}\right)$ is as large as possible. The chosen points are separated by a certain minimum distance (determined by the smallest element of $\Lambda$ ) and so the boundedness of $H$ forces the sequence to terminate, say at the point $x_{m}$.

We have shown that the sets $\left(K\left(x_{i}\right)\right)_{i=1}^{m}$ are disjoint, and it remains to show that the sets $\left(K_{2}\left(x_{i}\right)\right)_{i=1}^{m}$ cover $H$. Let $y$ be a point of $H$ which is not in any $K\left(x_{i}\right) i=1,2, \ldots, m . K(y)$ cannot be disjoint from all of $K\left(x_{1}\right), \ldots, K\left(x_{m}\right)$, or else it would be available as a choice for $x_{m+1}$. Let $x_{j}$ be the first point of the sequence $\left(x_{i}\right)_{i=1}^{m}$ for which $K\left(x_{i}\right) \cap K(y) \neq \varnothing$. Then $y$ was available at the $j$ th stage as a possible choice for $x_{j}$, and since it was not chosen, it follows that $\lambda\left(x_{j}\right) \geqq \lambda(y)$.

Part (i) now applies to show that $y \in K_{2}\left(x_{j}\right)$, and (iii) follows.

Proof of the main theorem. Suppose $c>0$ is given, and

$$
E=E(c)=\{x:(m f)(x)>c\} .
$$

The lower semi-continuity of $m f$ makes $E$ an open set, and in particular $E$ is measurable.

Let $H$ be a compact subset of $E$ and $\delta>0$ a positive constant; $H$ and $\delta$ are chosen independently of each other and of $c$.

For each $x \in H \supseteq E$, there will be a value of $\lambda$ for which $\left(\lambda^{n}|K|\right)^{-1} \int_{x+\lambda R} f>c$; furthermore, the easily verified fact that $\left(\lambda^{n}|K|\right)^{-1} \int_{x+\lambda K} f \rightarrow 0$ as $\lambda \rightarrow \infty$ shows that we may assume that $\left((2 \lambda)^{n}|K|\right)^{-1} \int_{x+2 \lambda K} f<c+\delta$. For each $x \in H$, we choose a value of $\lambda$ to satisfy both these properties. The continuity of the integral $\int_{x+\lambda K} f$ as a function of $x$ shows that there will be a neighbourhood $N(x)$ of $x$ such that the same value of $\lambda$ may be used throughout. We now use the compactness of $H$ to cover it by a finite number of neighbourhoods $N(x)$, and thus obtain a finite number of values of $\lambda$.

This brings us to the situation of (iii) of Lemma 2, which may now be applied to give a finite number of points $x_{1}, \ldots, x_{m}$ of $H$ with the properties that $\left(K\left(x_{i}\right)\right)_{i=1}^{m}$ are disjoint, while $\left(K_{2}\left(x_{i}\right)\right)_{i=1}^{m}$ cover $H$.

$$
\begin{aligned}
& \text { It follows that if } S=\bigcup_{i=1}^{m} K\left(x_{i}\right), \\
& \qquad|H| \leqq \sum_{i=1}^{m}\left|K_{2}\left(x_{i}\right)\right|=2^{n} \sum_{i=1}^{m}\left|K\left(x_{i}\right)\right|=2^{n}|S| .
\end{aligned}
$$


Also for each $x_{i}, \int_{K\left(x_{i}\right)} f>c\left|K\left(x_{i}\right)\right|$, so that on adding, we have $\int_{S} f>c|S|$.

With the aid of Lemma 1 (i), this may be rewritten

$$
0<c<\frac{1}{|S|} \int_{S} f \leqq \frac{1}{|S|} \int_{0}^{|S|} f^{*}=f^{* *}(|S|) \leqq f^{* *}\left(2^{-n}|H|\right)
$$

The fact that $f^{* *}(t) \rightarrow 0$ as $t \rightarrow \infty$ now shows that $|H|$ is bounded above. But $|E|=\sup \{|H|: H$ compact and $\subset E\}$, and so $|E|$ is finite, and

$$
0<c \leqq f^{* *}\left(2^{-n}|E|\right) \text {. }
$$

A corollary of this is that $\mid E\left\|/ 2^{n} \leqq \frac{1}{c} \int_{0}^{|E| / 2^{n}} f^{*} \leqq \frac{1}{c}\right\| f \|_{1}$ if $f$ is integrable. It follows from this inequality by a standard argument (see for instance (1)) that $\lim _{\lambda \rightarrow 0}\left(\lambda^{n}|K|\right)^{-1} \int_{x+\lambda K} f \rightarrow f(x)$ a.e., and in particular that $f(x) \leqq m f(x)$ a.e.

We may now prove inequality (i) of the theorem as follows.

Let $t_{1}>0$ be given, and let $c$ be any positive number with $0<c<(m f)^{*}\left(t_{1}\right)$.

Then $|E|=|\{x:(m f)(x)>c\}|=\left|\left\{t:(m f)^{*}(t)>c\right\}\right|$

$$
\geqq\left|\left\{t:(m f)^{*}(t) \geqq(m f)^{*}\left(t_{1}\right)\right\}\right| \geqq t_{1},
$$

where the facts that $(m f)^{*}$ is decreasing and equimeasurable with $m f$ have been used.

Inequality (iii) above now shows that $0<c \leqq f^{* *}\left(2^{-n} t_{1}\right)$, and since $c$ was any number less than $(m f)^{*}\left(t_{1}\right)$ we deduce that $(m f)^{*}\left(t_{1}\right) \leqq f^{* *}\left(2^{-n} t_{1}\right)$ as required.

In order to prove inequality (ii) of the theorem we again make use of the fact that the family $\left(K_{2}\left(x_{i}\right)_{i=1}^{m}\right.$ covers $H$. It follows that

$$
\int_{H} f \leqq \sum_{i=1}^{m} \int_{K_{2}\left(x_{1}\right)} f \leqq(c+\delta) \sum_{i=1}^{m}\left|K_{2}\left(x_{i}\right)\right|=\left(\frac{2}{\alpha}\right)^{n}(c+\delta) \sum_{i=1}^{m}\left|K_{\alpha}\left(x_{i}\right)\right|
$$

where $\alpha$ is a constant to be determined later. It will be necessary to restrict $\alpha$ to lie in $(0,1]$ in order to ensure the disjointness of the sets $\left(K_{a}\left(x_{i}\right)\right)_{i=1}^{m}$

We now show that each $K_{\alpha}\left(x_{i}\right)$ is contained in $E\left(c(1+\alpha)^{-n}\right)$. For according to (ii) of Lemma 2, if $y \in K_{\alpha}\left(x_{i}\right)$, then $G(y)=y+(1+\alpha) \lambda\left(x_{i}\right) K \supseteq K\left(x_{i}\right)$, and so we have

$$
|G(y)|^{-1} \int_{G(y)} f \geqq\left((1+\alpha)^{n}\left|K\left(x_{i}\right)\right|\right)^{-1} \int_{K\left(x_{1}\right)} f>c(1+\alpha)^{-n} .
$$

We can now deduce from inequality (iv) that

$$
\int_{H} f \leqq(2 / \alpha)^{n}(c+\delta)\left|E\left(c(1+\alpha)^{-n}\right)\right|
$$

and from the fact that $H, \delta$ were chosen independently it follows that

$$
\int_{E} f=\int_{E(c)} f \leqq(2 / \alpha)^{n} c\left|E\left(c(1+\alpha)^{-n}\right)\right| .
$$


Now let $A$ be any positive constant, and let $F$ be a measurable subset of $\mathbf{R}^{n}$ for which $A\left|E\left(c(1+\alpha)^{-n}\right)\right| \leqq|F|<\infty$.

Then

$$
\begin{aligned}
|F|^{-1} \int_{F} f & \leqq|F|^{-1} \int_{E} f+|F|^{-1} \int_{F \mid E} f \\
& \leqq\left(\frac{2}{\alpha}\right)^{n} \frac{c}{A}+\frac{|F| E \mid}{|F|} c \leqq\left(\left(\frac{2}{\alpha}\right)^{n} \frac{1}{A}+1\right) c,
\end{aligned}
$$

where we have used (v) and the fact that $f(x) \leqq m f(x) \leqq c$ at almost all points of $F \backslash E$. By (ii) of Lemma 1, it follows that

$$
f^{* *}\left(A\left|E\left(c(1+\alpha)^{-n}\right)\right|\right) \leqq\left(\left(\frac{2}{\alpha}\right)^{n} \frac{1}{A}+1\right) c .
$$

The proof of inequality (ii) of the theorem is now completed as follows.

Let $t_{1}>0$ be given, and let $c=(1+\alpha)^{n}(m f)^{*}\left(t_{1}\right)$. Then

$$
\left|E\left(c(1+\alpha)^{-n}\right)\right|=\left|\left\{x:(m f)(x)>(m f)^{*}\left(t_{1}\right)\right\}\right|=\mid\left\{t:(m f)^{*}(t)>(m f)^{*}\left(t_{1}\right) \mid \leqq t_{1} .\right.
$$

It follows that $f^{* *}\left(A t_{1}\right) \leqq f^{* *}\left(A\left|E\left(c(1+\alpha)^{-n}\right)\right|\right)$

$$
\leqq\left(\left(\frac{2}{\alpha}\right)^{n} \frac{1}{A}+1\right) c=\left(\left(\frac{2}{\alpha}\right)^{n} \frac{1}{A}+1\right)(1+\alpha)^{n}(m f)^{*}\left(t_{1}\right) .
$$

Inequality (ii) now follows on making the substitution $\alpha=1$ if $A<2^{n}$, or $\alpha=\left(2^{n} / A\right)^{1 /(n+1)}$ if $A \geqq 2^{n}$, and the proof is complete.

The choice of a particular value for $A$ is to some extent arbitrary: the value $A=2^{n}$ gives $2^{-n-1} f^{* *}\left(2^{n} t\right) \leqq(m f)^{*}(t)$ mentioned in the introduction, while the choice $A=1$ gives $f^{* *}(t) \leqq 2^{n}\left(2^{n}+1\right)(m f)^{*}(t)$ which is the estimate obtained in (2) and (6) when $K=Q_{n}$, the unit cube in $\mathbf{R}^{n}$.

The fact that if $f$ is essentially bounded, $\lim _{t \rightarrow 0}(m f)^{*}(t)=\lim _{t \rightarrow 0} f^{* *}(t)=\|f\|_{\infty}$ (which is also evident from elementary considerations) may be deduced from (i) and (ii) by taking $A$ large.

\section{Further results}

For a given $K$, we denote by $\eta(K)$ the smallest constant for which

$$
(m f)^{*}(t) \leqq f^{* *}(t / \eta(K))
$$

for all $f \in L^{*}\left(\mathbf{R}^{n}\right)$ and ${ }^{-} t>0$.

With this notation, (i) of Section 2 can now be stated simply as

$$
\eta(K) \leqq 2^{n} \text { for allysuitable } K \subset \mathbf{R}^{n} \text {. }
$$

We denote the unit sphere and unit cube in $\mathbf{R}^{n}$ by $K_{n}$ and $Q_{n}$ respectively. We can now make the following observations concerning the size of $\eta(K)$.

(1) $\eta(K)>1$. For the value of $\eta(K)$ is independent of rotations or dilations of $K$, and so without loss of generality we may assume that $\frac{1}{2} \operatorname{diam}(K)=1$ 
and that $e_{1}=(1,0, \ldots, 0)$ is a boundary point of $K$. Consider now a unit mass placed at each of the points $\left(r e_{1}\right)_{r=-N}^{N}$. (Such a mass-distribution is a limit in an obvious sense of a sequence of functions in $L^{*}\left(\mathbf{R}^{n}\right)$ whose supports shrink to the points $r e_{1}$, while their integrals remain constant.) If we take $\varepsilon_{n}=\left(1+2^{1 / n}\right)^{-1}<\frac{1}{2}$, an easy calculation shows that $m f(x) \geqq\left(\varepsilon_{n}^{n}|K|\right)^{-1}$ at all points of $r e_{1}+\varepsilon_{n} K,(r=-N, \ldots, N)$ and also at all points in the "overlap" $\left((r-1) e_{1}+\left(1-\varepsilon_{n}\right) K\right) \cap\left(r e_{1}+\left(1-\varepsilon_{n}\right) K\right)$. Hence if $\mu_{n}$ denotes the measure of this last set, we have $m f(x) \geqq\left(\varepsilon_{n}^{n}|K|\right)^{-1}$ on a set whose measure is at least $(2 N+1) \varepsilon_{n}^{n}|K|+2 N \mu_{n}=\tau$ say. It follows that $(m f)^{*}(\tau) \geqq\left(\varepsilon_{n}^{n}|K|\right)^{-1}$. But $f^{* *}(t)$ is simply $(2 N+1) t^{-1}$ for all $t>0$, and so

$$
1 \geqq \frac{(m f)^{*}(\tau)}{f^{* *}(\tau / \eta(K))} \geqq \frac{\left(\varepsilon_{n}^{n}|K|\right)^{-1}}{2 N+1} \cdot \frac{\tau}{\eta(K)}
$$

or

$$
\eta(K) \geqq 1+\frac{2 N}{2 N+1} \frac{\mu_{n}}{\varepsilon_{n}^{n}|K|} .
$$

But $N$ may be taken arbitrarily large, and so $\eta(K) \geqq 1+\mu_{n}\left(\varepsilon_{n}^{n}|K|\right)^{-1}>1$. In the case $K=K_{n}$ the estimate obtained tends rapidly to 1 for large $n$.

(2) $\eta\left(Q_{1}\right)=\eta\left(K_{1}\right) \geqq \frac{3}{2}$. This is simply the case $n=1$ of the above, when $K=(-1,1), \varepsilon_{n}=\mu_{n}=\frac{1}{3}$. Thus we have a gap, even in the one-dimensional case between the upper estimate $\left(\eta\left(K_{1}\right) \leqq 2^{1}=2\right)$ given by the theorem, and the lower estimate $\eta\left(K_{1}\right) \geqq \frac{3}{2}$.

(3) $\eta\left(Q_{n}\right)$ is an increasing function of $n$. For if $f(x)=f\left(x_{1}, x_{2}, \ldots, x_{n}\right)$ is a function on $\mathbf{R}^{n}$, which gives a near approximation to $\eta\left(Q_{n}\right)$, then $f^{\sim}\left(x_{1}, x_{2}, \ldots, x_{n}, x_{n+1}\right)=f\left(x_{1}, x_{2}, \ldots, x_{n}\right)$ gives the same approximation for $\eta\left(Q_{n+1}\right)$. The details are identical to those in Section 3 of (6).

(4) $\eta\left(K_{2}\right) \geqq \frac{3}{2} \frac{\sqrt{2}+\sqrt{3}}{\pi}>\frac{3}{2}$. This estimate is obtained by considering a hexagonal lattice of unit masses in the plane: we outline the argument briefly.

Consider unit masses at the points of the lattice generated by the points $P_{1}=(1,0)$ and $P_{2}=\left(\frac{1}{2}, \sqrt{3} / 2\right)$. If we restrict attention to a large area $V$ of the plane, there are approximately $2 V / \sqrt{3}$ point masses, and so $f^{* *}(t)=2 V /(\sqrt{3} t)$. Let the point $P$ be determined by

$$
\left(\pi(O P)^{2}\right)^{-1}=2\left(\pi\left(P_{1} P\right)^{2}\right)^{-1}=3\left(\pi\left(P_{2} P\right)^{2}\right)^{-1}=\rho
$$

say. It is easily verified that $P$ is $(1 / \sqrt{6},(\sqrt{3}-\sqrt{2}) / \sqrt{6})$ and that the corresponding value of $\rho$ is $\left(\pi\left(1-\sqrt{\frac{2}{3}}\right)\right)^{-1}$. Then at each point of the triangle $O P_{1} P_{2}$, $m f$ has a value at least as large as $\rho$, and so $m f(x) \geqq \rho$ for all $x \in V$.

Hence $(m f)^{*}(V) \geqq \rho$, and so

$$
1 \geqq \frac{(m f)^{*}(V)}{f^{* *}\left(V / \eta\left(K_{2}\right)\right)} \geqq \rho / \frac{2}{\sqrt{3}} \eta\left(K_{2}\right) .
$$


Hence

$$
\eta\left(K_{2}\right) \geqq \frac{\sqrt{3 \rho}}{2}=\frac{\sqrt{3}}{2} \frac{1}{\pi\left(1-\sqrt{\frac{3}{2}}\right)}=\frac{3}{2} \frac{1}{\pi(\sqrt{3}-\sqrt{2})}=\frac{3}{2} \frac{\sqrt{3}+\sqrt{2}}{\pi}>\frac{3}{2} .
$$

(5) The estimate $(m f)^{*}(t) \leqq f^{* *}(t / \eta(K)) \leqq f^{* *}\left(2^{-n} t\right)$ leads at once via Hardy's inequality (see (5) page 196) to a norm estimate for $m f$ if $f \in L^{p}\left(\mathbf{R}^{n}\right), 1<p<\infty$. Thus

$$
\begin{aligned}
\|m f\|_{p} & =\left\|(m f)^{*}\right\|_{p} \leqq\left\|f^{* *}(. / \eta(K))\right\|_{p} \leqq(\eta(K))^{1 / p}\left\|f^{* *}\right\|_{p} \\
& \leqq(\eta(K))^{1 / p} p /(p-1)\left\|f^{*}\right\|_{p}=(\eta(K))^{1 / p} p /(p-1)\|f\|_{p} .
\end{aligned}
$$

In particular $\|m f\|_{p} \leqq 2^{n / p} p /(p-1)\|f\|_{p}$.

The following example gives some information about the constants which appear in inequality (ii) of Section 2.

(6) Let $f(x)=|x|^{-\alpha}$, where $|x|^{2}=x_{1}+x_{2}^{2}+\ldots+x_{n}^{2}$, and $0<\alpha<n$.

Then if $0<\alpha \leqq n-2, f$ is a superharmonic function on $\mathbf{R}^{n}$, so that

$$
A(f, x, \lambda)=\left(\lambda^{n}\left|K_{n}\right|\right)^{-1} \int_{x+\lambda K_{n}}
$$

is a non-increasing function of $\lambda>0$. In particular $(m f)(x)=f(x)=|x|^{-\alpha}$, and so

$$
(m f)^{*}(t)=\left(t /\left|K_{n}\right|\right)^{-\alpha / n}=f^{*}(t)=\frac{n-\alpha}{n} f^{* *}(t),
$$

and when $\alpha=n-2,(m f)^{*}(t) / f^{* *}(t)$ has the constant value $2 / n$.

\section{REFERENCES}

(1) M. de Guzmán and G. V. Welland, On the differentiation of integrals, Rev. Un. Math. Argentina, 25 (1971).

(2) C. S. Herz, The Hardy-Littlewood Maximal Theorem, Symposium in Harmonic Analysis (University of Warwick, 1968).

(3) F. Rresz, Sur un théorèm de maximum de MM Hardy et Littlewood, J. London Math. Soc. 7 (1931), 10-13.

(4) E. M. StEIN, Singular integrals and differentiability properties of functions (Princeton, 1970).

(5) E. M. STEIN and G. WeIss, Introduction to harmonic analysis on Euclidean spaces (Princeton, 1971).

(6) P. L. WALKER, Some estimates for the Hardy-Littlewood maximal function in $R^{n}$, Bull. London Math. Soc. 7 (1975), 139-143.

UNIVERSITY OF LANCASTER

$$
\text { E.M.S. }-19 / 4-2 B
$$

